\title{
The Matching of the Scholar Projecting and Perspective Planning in Russian Musical Communication
}

\author{
Alexander Yakoupov \\ Russian State Specialized Arts Academy \\ Moscow, Russia
}

\begin{abstract}
The article is dedicated to the consideration of necessities of the scholar's approach towards the musical communications management. The author underlines the role of the qualitative planning method of social cultural life. The examination of musical activity in spheres of Russian culture is presented in this paper.
\end{abstract}

Keywords-musical culture; planning of music communication; music development of person; the qualitative criteria

\section{INTRODUCTION}

The scientific methods of management traditionally imply that creating a programme for the development of a given social sphere is followed by working out particular plans for realising the programme elements - the plans that can be both long-term (perspective) and short-term (operational).

In this country, for a long time, the content of the social plans for developing musical culture was characterised by an extensive quality. The management and administration objectives, although presented as complex, actually proved to be one-dimensional and rather primitive since the planning was mostly based on quantitative data.

The approach to planning was quite trivial: the financial and material means were distributed among all the institutions of the musical culture, irrespective of their actual requirements, that is, according to the so called 'average' norms. The general staff schedules for the musical personnel, the unified wage schemes, the norms of expenditure on the upkeep of the buildings, buying the instruments, repairs and renovations, and etc. were drafted and approved. For instance, the classes for a conservatoire student were planned according to the identical norm, no matter whether a student was preparing to take part in a performance contest or not, whether he lived in a hostel or a boarding house. The development of the material base of the musical art was conducted in the analogous way, that is, the building of the opera theatres, the philharmonics, creating of the musical education institutions, colleges and schools, organising performing collectives (orchestras, choirs, etc).

\section{The PeCUliarities OF THE PlanNING IN THE SPHERE OF ART}

We have to note here that, in the 60s, the situation in the musical life changed perceptibly. The conditions were created for a musical life to take on a certain cyclic character. The tendency of making the musical values open and available to the public was not properly reflected in the principles of planning in the sphere of art - the planning still remained extensive. It is not accidental since the other, intensive, way of development and the appropriate approach to planning are unthinkable without using certain theoretically justified principles. Unfortunately, the theory of management and administration in the sphere of art constantly lags behind the existing social requirements. Consequently, the planning related to the optimisation of the processes of musical communication that form the very basis of the musical life - the planning was invariably based upon the outdated principles of the same extensive method.

One of the outcomes of the cited approach towards planning the musical life of the Russian cities - one of the outcomes of such approach was contained in the stable tendency to create the social institutes of the appropriate kind of art only in the major administrative centres. The other urban entities, even those possessing a great potential in the various spheres of culture taken broadly - in the economic, industrial, educational sphere - such urban centres were only given secondary importance in relation to the social being of the serious music.

In other words, the centralised approach, in spite of all the criticism, has failed to take into consideration the local peculiarities and initiatives. The later opportunity for the art institutions to be transferred from federal into municipal proprietorship had not changed the situation much since the local authorities lacked funds for expanding the network of such institutions and reorganising the existing ones. Even the local traditions in this field were hardly given proper attention. It is astonishing that in the national cultural practice, until just recently, there has not been a precedent (indeed, there hardly is now) - a precedent when, say an opera theatre, a conservatoire, a philharmonic society, or a musical publishing house was established in a provincial town. 
The principles of uniformed and centralised planning were reflected in the approach to musical education development. Thus, the uniformity of the curricula in the system 'elementary musical school - college - conservatoire' has led to a considerable decline in the number of those willing to study at the elementary and secondary musical schools. According to the expert estimates by specialist pianists, violinists and 'cellists in 1992, the number of such students in central Russia decreased three, in some cases four, times as compared to 1970. Thus, the very basis for developing the processes of musical communication is eroded - the basis that invariably depends on the educational factors existing in all the spheres of communication.

The principle of planning according to what has already been achieved established in the economy, if used in the sphere of music, could only augment the existing conflicts and discrepancies. The principle is based upon investing at the year-before level, its utilisation proves to be a means of limiting the musicians' creative initiative, their opportunity to extend the field of the socio-musical communication, to stimulate its actual development. Therefore, the musical life (and the aesthetic sphere in general) came to be dominated by the so-called 'method of spontaneous proportions' (Y. U. Focht-Babushkin).

The administrative structures' inability to localise, by means of effective, scientifically justified planning, the disintegration of such areas of musical life and culture as the musicology, musical performing, education and upbringing, the musical psychology, sociology and cultural theory - this inability also produces its negative impact.

Each of the cited areas has accumulated significant theoretical potential, but those areas, as a rule, exist without proper interconnection. One may conclude that the communicative links between them - the links belonging to the meta-structural level - are obviously quite inadequate.

For instance, the vast information on the regularities of human musical communication, the specific character of the language of music, the stylistic peculiarities of the certain composers' creativity and works, the compositional characteristics of different epochs, styles and genres - all these data are not properly utilised by the performers. Still, without such knowledge, an adequate understanding of the performed pieces' deeper meanings is quite problematic. Thus, the flow of musical information that an interpreter is called upon to give over to the listener is substantially limited.

We would also like to note a very important aspect here. As a rule, the specialists in musical education lack familiarity with the sociology of music. Consequently, they cannot properly understand the development of the musical culture. So, it is not accidental that the music school graduates eventually reject serious music. Oftentimes, the particular reasons for this phenomenon are contained in the neglect of the principles of the educational repertoire selection, the lack of consideration towards each student's taste, towards the specific regional and ethnic qualities of the musical culture development.
All that has led to serious mistakes in building the national musical culture has, furthermore, led to major decrease in the number of the potential listeners of the academic genres of music. The whole complex of reasons that account for the general fall of interest towards the traditional forms of the spiritually charged music is much more diverse and complicated. These reasons are largely connected to the global changes in the social, cultural and economic development, the socio-psychological condition of the different social groups. But the absence of the theoretically supported and reliable principles and methods of planning produces its negative impact. So, the administrators are often right to blame the specialist musicologists because they fail to provide the necessary methods and techniques for planning the administrative activity in the complex and very specific sphere of the musical art.

There are serious reasons for all that. The intensive way which the planning of the musical culture development should follow implies a certain degree of knowledge of the more subtle regularities that characterise the processes of musical communication, the regularities related to the deeper factors of the person's inner world, and to the existence of the musical piece in the complex conditions of the multichannel chain of musical communication that implies a multiplicity of variants of the individual music perception. All that increases the complexity of administration, since the quality of the appropriate processes in the society mostly depends exactly upon the qualitative, not quantitative characteristics - the qualitative characteristics that are hardly ever pronounced in the external attributes of the plans.

One should note that a number of issues are still unclear to the theorists themselves. Today, only the first steps are made towards the realisation of the problems of musical communication, the most important tasks are singled out, approaches to the solution of the problems are searched for. The most serious conflict that exists in the present-day aesthetic-based life of a society is between 'the great functional capabilities of music in moulding the personality and the little time that the people can allot to the contact with art' [1, P. 176-177]. The studies by L. A. Gordon, E.V. Klopov and L.A.Onikov discovered that, say, a worker only spends an average one hour a day on some kind of activity related to art and aesthetics (including watching television). Taking that into consideration Y. U. Focht-Babushkin suggests that the time should be rationally organised and planned in order to be used more effectively.

Still, the analysts believe that the task is not that easy, since the levels of human musical development are not yet quite established in the national and foreign musicology, the criteria for the degree to which the musical knowledge is absorbed are not specified. The regularities of accumulating the communicative experience, and the skills and techniques related to music are not thoroughly researched. The mechanism of co-operation between the personality's musical development and other kinds of aesthetic education 
is not yet thoroughly elaborated *. All that presents the theorists and scholars working in the field of planning the musical life with a number of major problems.

Still, recently there have appeared several studies that testify to the fact that the attempts to develop the appropriate sets of methods persist [see $2 ; 3$ ]. Their peculiarity is the orientation towards the local planning of the musical life and the subjects' of communication activity in a given community, educational institution, a professional group, etc. [see 2]. The current and perspective planning is usually based in this case upon a sufficiently adequate study of the musical needs of a particular social community. Nevertheless, the majority of such concepts are unfortunately based on the traditional enlightenment idea that consists in providing a wider access to the real musical values in order to satisfy the appropriate requirements, as if they already exist. Plus, the task is often simplified and reduced to making the contacts of the listeners with the serious music as frequent as possible.

\section{THE Problems of Human ARTS DEVElopment}

L. S. Vygotsky and his followers have proven that the process of the human aesthetic development is most effective when the person absorbs art and aesthetic creativity directly and integrally, through perceiving its products, gaining the knowledge related to them and actually taking part in some creative activity.

It is also noted that each age period calls for a stress upon those genres and kinds of the personal creative activity that provide for the greatest impact upon the human being exactly at this stage of his personal evolution. Attempts were made to find the optimal points of coming in contact with the art, the best scale and degree of its consumption. We would try to consider the issue in a slightly greater detail since it is one of the major problems of planning in the sphere of music.

A number of theorists believe that there is no need for defining the norms of 'consuming' the high aesthetic values of the musical art, since the human development is unlimited in principle. There is a comparison to the entertainment music forwarded as an argument. Thus, M. Sushchenko calculated that this kind of music is broadcast in the different radio programmes for up to 80 hours a day ${ }^{*}$. The comparison is not in favour of the serious music.

Some theorists, on the other hand, believe that there should be some optimal levels and degrees of coming into contact with the musical values. A. Moles believes that such contact should not be unlimited or excessive, and that the very limitations of the human perceptive channels (the hearing and vision) serve as an argument to support such a view [4, P. 130].

Using the results of a study by A. Y. Vakhmetsa and S. N. Plotnikov, Y. U. Focht-Babushkin even suggests that the

\footnotetext{
* It is noteworthy that these issues are now addressed by the culture theorists, especially those who are practically connected with training musicians, particularly pedagogue musicians (see, for instance, 246).

* The data as of 1979 . With the introduction of new channels the duration of transmitting music through the radio communicative channels has increased greatly.
}

excessively frequent contact with a work of art may lead '... to a perceptible deterioration in the quality of preferences' [1, P. 185]. Thus, some theorists are making attempts to establish the optimal quantity of the contacts with serious music for the school students of different age - from two to nine concerts a year [see 1, P. 187].

M. Sushchenko has expressed a similar opinion, though in a slightly different aspect [see 5]. Arguing with V. S. Zuckerman in respect of the certain methods of evaluating the musical preferences of the public that the latter had suggested, Sushchenko wrote, 'One might even imagine the reverse: the more dear and valuable a work of art is to a person, the more he tends to 'save' it for himself, avoid exhausting it, since when a piece sounds much too often, it loses its importance, becomes hackneyed, commonplace and unnoticed, even if the initial impression was very strong. Without a doubt, true love and appreciation of art does not equal a constant contact with it' [5, P. 252].

Still, we believe that this kind of approach draws certain objections. One should think that speaking of the constant contact the author does not imply anything quite literal (say, listening to music around the clock). He rather means the danger of being surfeited with similar aesthetic impressions. But one should beware any forced contacts, rather than just frequent ones.

The psychologists have noted that human rejection of anything (including something pleasant or beneficial, etc) is directly dependent upon the degree of violence or compulsion that the human being suffers. Sometimes a single contact with a work of art is sufficient to 'devalue' it completely for that particular person. But when the individual is free from compulsion, there is no concern of this kind for an emancipated mind is quite a guarantee against possible surfeit.

The tendency to 'save' the most valuable could be considered had the world of art possessed a single musical work of supreme aesthetic significance. Fortunately, it is quite impossible to devalue the whole musical universe since the musical values accumulated by the mankind are quite countless.

Coming back to the issue of optimal levels, we should note that the theorists have previously emphasised just the quantitative criteria. We believe this to be a certain indication that the method they used is a little bit limited. The serious music includes the pieces (symphonies, cantatas, oratorios, operas, instrumental concertos) that are much more complex and difficult for perception than, say, the miniatures from the children's albums by some authors, the small instrumental pieces used in the course of musical education or for household performance. Neither the genre, nor the stylistic criterion acts as a factor of planning in the suggested method. The relationship of the utilised genres and the children's age peculiarities is not considered. One can demonstrate some other considerable drawbacks in the method in question.

For instance, A. L. Vakhmetsa and S. N. Plotnikov failed to establish the reasons for the change in the quality of the 
preferences. They only stated that the higher level of the preferences in the motion-picture art is characteristic of the viewers who watch films regularly - within the average norm ( 30 to 50 pictures a year, that is, 2 - 4 times a month), and the lower level was manifested by the viewers who are either too infrequent (less than 10 times a year) or too habitual watchers ( $7-8$ times a month).

We believe that such a statement cannot serve as a demonstration of the relationship between the level of the preferences and the quantity of contacts with the cinema. Following this logic, one might assume that the people who go to the movies 'too seldom' or 'too often' are potentially incapable of demonstrating a different quality of preferences, even if they try to attain the optimal norms of 'consumption'.

The aforementioned theorists have neglected the obvious dependence of the preferences upon the aesthetic quality of the pictures themselves. We should note here that the cited work was published in 1968. It was the time when the theatres played the significant works by Tarkovsky, Khutsiev, Bondarchuk, as well as the Indian melodramas and the first American action movies to be shown in Russia. Which kind of the pictures formed the recipients' preferences had unfortunately remained unclear.

We could only add one consideration. Following the suggestion that excessive 'consumption' of the aesthetic values leads to a quality deterioration in preferences one might come to the conclusion that all the professional musicians, actors, artists, poets etc are in danger of acquiring a very doubtful taste because of an abundant 'consumption' of the aesthetic values related to their professional activity.

Taking all the above into consideration, we would like to make a number of conclusions. First, we would like to state that the related theory of today offers no obvious proof that there is a certain maximum norm beyond which a sharp deterioration in the quality of the preferences takes place.

Second, we would have to concede that establishing of the optimal top level in the contacts with music (even if it actually had to be done) is quite impossible for the simple reason that the different social groups consist of actual people - unique personalities - with their invariable wish to satisfy their individual needs. One should not forget here that in the social life there is not, and there can't be, a mechanism to contain or restrict the man's inner drive for self-expression that is most obviously manifested in art.

Third, and finally, we believe that setting a certain norm for the contacts with the musical art, finding a most rational extent of such contacts and their limiting maximum level cannot be a really acute problem today since even the most indispensable minimum is now hardly achieved. Thus, from the organisational viewpoint, the attempt to limit the contact with serious music has no real significance in the discussion of the related theoretical subjects.

We believe that it is worthwhile rather to define the minimum level without attaining which the development of a spiritually mature individual is quite problematic, as well as the formation of his musical and aesthetic taste. The period during which the interest to certain music wanes after the last contact with it may serve as the criterion for establishing such a minimum. We believe that one of the major aims of the appropriate management is contained in the idea to prevent the waning of interest towards the piece recently heard. Therefore, this idea may serve as an important objective in planning the development of the social processes of musical communication.

Planning of the network of musical culture institutions, as well as of the contact with and assimilating the aesthetic values is impossible without taking into consideration the appropriate public needs and requirements and forecasting their perspective formation. To clarify the present condition of those needs is difficult but possible. What is harder is to forecast their future development.

The national practical experience normally utilises three ways of projecting the future tendencies. The first and the most widely established method is based upon the analysis of the tendencies and results of the previous period. Taking into consideration the drawbacks of this method, the theorists justly note its inadequacy in respect of the interest formation. It is meant here that 'the recently established situation in the population's contact with art, all its preferences and handicaps are, for no particular reason, set as the goals for the year ahead. Besides, this particular method focuses upon satisfying the needs, not forming them for it implies following the emerging tendency rather than influencing it' [1, P. 197].

The second method of forecasting is known as the 'expert poll'. This method, based upon studying the authoritative specialists' opinions, is at once attractive and largely imperfect. The fact that the appropriate theory still lacks sufficiently reliable objective knowledge in the sphere of art has to be taken into consideration. The expert opinions and forecasts, quite often based on intuition, are often rather contradictory. Therefore, selecting the solutions suggested by the scholars the administrators face a considerable risk of making a mistake. Working in the same sphere, the experts oftentimes present their private interests as the objective tendency. In short, the whole matter can be really too subjective, subtle and complex.

The third method is realised by setting certain eventual characteristics. Y. U. Focht-Babushkin believes it to be the most effective. It is essentially contained in elaborating certain significant characteristics that are formed in relation to the goal that the society sets its culture. The aspect of an actual opportunity to manage the processes of musical communication is very attractive in this whole method.

Still, there are certain difficulties here, too. In case the method is utilised by unable and incompetent administrators who are ready to achieve their aims at whatever cost there is very little that the real art could gain by it. Plus, it is very hard to develop a model that would essentially correspond to the public intentions.

Such a model of the desired future may be contained in the generalised profiles of the more culturally developed territories, organisations, educational institutions, etc. This way of planning is usually termed as the 'leading groups' of 
the population method'. Favouring its use in principle, Y. U. Focht-Babushkin believes that since the sociologists utilise the method to research the 'social requirements'. It would be more appropriate to discuss 'the socially developed' groups of the population, not the 'leading' ones. The 'socially developed' groups of the population are traditionally believed to include the industrial and agricultural workers, the intelligentsia, that is, the social layers that demonstrate the traits of a more perfect way of life. Using the approach Y. U. Focht-Babushkin concluded that '...the contacts with art among the 'socially developed' people are generally more frequent than the national average. Therefore the frequency with which the 'socially developed' people visit the cultural institutions would not be excessive, but will be at once considerably higher than average and so may serve as the direction in the development of the network of the cultural institutions. The contacts that the 'socially developed' people have with art may thus serve as the example of the opportunity that the population should be given respect of the contact with art' [1, P. 199].

\section{The Practice of PlanNing IN THE SPHERE OF MUSIC}

The detailed analysis of the methods and techniques of planning developed in the culture theory was important for this particular study of the musical communication because an analysis of the kind may be important to define the degree to which the cited methods and techniques may be used in the sphere of music.

The phenomenon of the preference polarisation shows itself quite particularly in the musical sphere. That which is acceptable in, say, cinema or painting may be inadmissible in music. A certain discrepancy between the general planning methods and the goals of the musical life development are quite easy to demonstrate. For instance, the culture theorists claim that an excessive contact with art leads to the situation when the aesthetic values are devalued for the particular individual whose requirements are quite surfeited. This kind of forecast does not seem to justify itself in case of the entertainment music. The forecasted surfeit does not take place even after listening to it for almost six hours a day*

It has been noted that sometimes serious music, even sounding in the background, irritates the people who are not at all bothered by the same background of, say, entertainment music. Plus, in the latter case the demand usually exceeds the supply, whereas the situation with serious music is quite different. The high musical art is only really accepted by a small percentage of the population.

It is important to say that the musicians note that in the course of contact with this art the 'supply' really plays the

\footnotetext{
* It is the amount of time during which, according to sociologists, a human being finds himself in contact with the light music daily (including the music from motion pictures, radio and TV broadcasts, sports programmes, weather forecasts, etc). Moreover, the amount has probably grown by today due to the increased use of music in the different kinds of advertisement and information presentation. We should also remember the fact that certain (young) people use headphones to make the sound background their constant life companion.
}

major part. It is obviously no point to try and compete for popularity with the entertainment genres. Composers A. Schnitke and S. Slonimsky expressed the idea - they wrote that 'the serious music never tried to compete with the light music, including the competition for mass popularity, it has always attempted to find its own, deeper and more complex area of existence' [6, P. 25].

It is this 'deeper area of existence' that is able to impact the functioning of the musical communicative structures that the culture theory methods of planning fail to consider, since the methods still lack the criteria for the perspective planning of the development of the significant moments of the musical culture. Therefore, the methods are still largely reliant upon the quantitative characteristics.

In order to verify the conclusions, we have conducted an experiment. Its chief object was to develop the target quantitative levels of the general public's concert visits in a large industrial town ${ }^{*}$.

It is important to note that from the very start of the experiment the city's musicians realised that this particular planning method is most effective for seeing the drawbacks and handicaps of the existence of music in the present day society. As for the possibility to define the perspective tendencies, the quantitative data that had been received (according to the leading group formula) were quite obviously rather indefinite. Thus the data failed to produce the impression that the work conducted on the basis of this planning method is sufficiently useful and important.

It was also very difficult to define who the leading groups actually consisted of. The attempts to find those people by polling the employees at the different companies and organisations had failed (the cultural committees of the local labour organisations were polled). Almost everywhere the suggested candidates were only characterised by the two standard formulas - 'a great worker' and 'a wonderful person'.

There turned out to be a necessity of working out the criteria to select 'the socially developed persons'. The characteristics of those people should imply an evaluation of the following: the quality of the professional and social activity, the relationships within the group/collective (including the proof of the group's trust and respect - being elected to some social and labour organisations, different councils, etc), the presence of some kind of a musical hobby, and etc. For the students, the poll was conducted during a classroom discussion when the candidates for the leading groups were nominated. When such groups were formed, their actual participation could not leave one assured that those were 'the people of the future' whose lifestyle should be observed and followed.

There are several reasons for those doubts. For instance, the polls among adults have proven that in spite of their understanding the spiritual importance of the deeply meaningful musical art and their 'respectful attitude towards

\footnotetext{
* The experiment was conducted in 1982 in Magnitogorsk under the general guidance of the present author.
} 
it' many respondents were unable to specify their favourite classical genres, recognise the well-known pieces and name their authors (the test included 10 fragments from the very basic concert and opera repertoire that should be familiar to every schoolchild).

In course of the very experiment, in order to compare the various data, along with the experimental groups, two other control groups were formed: a group of experts consisting of specialist musicians and a group of the real music lovers. The results were devastating. Say, in the course of defining the future trends according to the first method it was found that every adult from 'the socially developed people' group visited an average of 0.8 concerts a year during the recent three years, the music lovers visited 10, and the specialists set 'a record' of three concerts a year. The administrators rejected the idea of forming a certain average level based on the given research data.

Then, the selection method was complicated. It came to contain the significant, the qualitative criteria. The principle that we developed postulated that the levels may be established based on the results of a special test containing $a$ set of purposeful suggestions.

The appropriate suggestions were organised in tables that contained the following:

- the titles of the various academic musical forms and genres (the instrumental chamber and orchestral music; then - operas, ballets; the vocal and choral music);

- the titles of certain genres of the folk instrumental music, vocal folklore;

- the names of the famous musicians - performers;

- the names of the prominent composers of the past and present;

- the titles of the particular musical works taken from the current itineraries of the various concert organisations for a given period. The questionnaire complex also contained one blank form which had a supplement of a long list of pieces from, so to speak, 'the golden fund of the world musical culture'.

According to the suggestions that the recipients made, the qualitative preference characteristics for the various groups 'consuming' the serious music were established. And from the initial planning stage of discussing the particular suggestions (the definite pieces, genres, the performers' names, etc) it became obvious that the participants of the experiment were obviously willing to increase the originally suggested minimum concert 'quota'.

The tendency for the significant and the content part of the plans to dominate over the quantitative ones remained stable during the five years of developing and realising the appropriate plans. Thus, the high quality of performing the pieces included into the plans greatly stimulated the listeners' activity.
In the course of the five-year period the minimums set for the contact with the serious music were usually exceeded by those listeners' groups to which the concerts were addressed. There was no surfeit with music perceptible (at least not on the group level). The decrease in the number of listeners only took place when there was a failure to provide the gradually improving quality level - both from the content viewpoint and the aesthetic level of performance.

\section{CONCLUSION}

The planning of the contacts with the musical values and stimulating the processes of the social musical communication is quite justified and rather necessary. At the same time, such planning is only possible if it is based upon the undoubted priority of the content (the qualitative) criteria. The quantitative characteristics and measurements in this procedure are directly related and dependent upon the content, the qualitative ones. Therefore, they are in no position to assume the leading role.

\section{REFERENCES}

[1] FOCHT-BABUSHKIN, Y. U. The Aesthetic Culture: The Issues of Study and Administration. - M., 1986.

[2] The Aesthetic Education of the Young: Problems and Prospects / Compiled and edited by M. M. Berlyanchik. - Novosibirsk, 1989.

[3] The Aesthetic Needs and Their Development in the Young: An Attempt of the Socio-aesthetic Study of the Students' Aesthetic Needs / Compiled by A. N. Semashko. - Kiev, 1970.

[4] MOLES, A. The Theory of Information and the Aesthetic Perception. - Translated from French. - M., 1966.

[5] SUSHCHENKO, M. O. Upon Certain Problems of the Detailed Sociological Study of Music in the USSR // The Issues of the Sociology of Art. - M., 1979. - P. 240 - 272.

[6] SCHNITKE, A., SLONIMSKY, S. The Glance from the Past Decade // Musical Academy. - 1992. - \# 1. - P. 20 - 26. 